\title{
PRODUÇÃO DE ÉSTERES ETÍLICOS DERIVADOS DE ÓLEO DE FRUTO DE MACAÚBA EMPREGANDO TECNOLOGIA DE ULTRASSOM
}

\author{
R. P. SCHERER ${ }^{1}$, E. MORESCO ${ }^{1}$; N. L. D. NYARI ${ }^{1}$; C. DALLA ROSA ${ }^{1}$; \\ D. de OLIVEIRA ${ }^{2} ;$ J. V. de OLIVEIRA ${ }^{2}$ \\ ${ }^{1}$ Departamento de Engenharia de Alimentos, URI - Campus de Erechim, \\ CEP: 99700-000, Erechim, RS. \\ 2 Departamento de Engenharia de Alimentos UFSC, CEP:88040-900, Florianópolis, SC. \\ rquimico@gmail.com
}

\begin{abstract}
RESUMO - Este trabalho visa na produção enzimática de ésteres etílicos em ultrassom nos modos batelada e contínuo empregando a lípase comercial Novozym 435 e óleo de macaúba (Acrocomia aculeata). As condições experimentais empregadas no estudo foram: razão molar óleo/etanol $1: 9,65^{\circ} \mathrm{C}$, $20 \%(\mathrm{~m} / \mathrm{m})$ de enzima, potências de 0 e 132 Watts, para os modos batelada e contínuo. Para modo continuo usou-se vazão de $2,5 \mathrm{~mL} / \mathrm{min}$. Verificou-se que a potência do ultrassom em ambos os modos estudados (batelada e contínuo) apresentaram influência positiva na conversão em ésteres etílicos com conversões em torno de $78 \%$ em tempo reacional de 30 minutos (modo batelada) e $69 \%$ em 11 minutos (modo contínuo).
\end{abstract}

\section{INTRODUÇÃO}

A busca por fontes alternativas de energias renováveis devido à possibilidade de escassez da oferta de combustíveis fósseis e a crescente preocupação com os impactos ambientais decorrente da emissão de materiais poluentes que provém da exaustão dos combustíveis derivados do petróleo, tem levado a pesquisas sobre diferentes fontes de combustíveis alternativos. Dentre esses combustíveis, o biodiesel se destaca em virtude do seu elevado potencial como substituto dos combustíveis fósseis, uma vez que pode ser usado puro ou na forma de misturas com óleo diesel (Demirbas, 2007; Gog et al., 2012).

A produção de biodiesel apresenta-se hoje como uma solução ambientalmente correta e significativa na geração de energia limpa. A transesterificação de fontes oleaginosas conduz à diminuição da viscosidade do óleo vegetal melhorando assim o desempenho do mesmo em motores movidos a diesel. Atualmente, o biodiesel é obtido através de reações de transesterificação entre um óleo vegetal e um álcool de cadeia curta na presença de catalisador. (Demirbas, 2007). Atualmente, 90\% do biodiesel produzido no país é obtido a partir do óleo extraído da soja virgem. Com a crescente demanda da produção de biodiesel visando esta ser uma tecnologia limpa e renovável, a busca por fontes alternativas produtoras de óleos que venham a complementar a cadeia produtiva do biodiesel se faz necessária (Biodiesel BR, 2013). 
Uma fonte promissora para produção de biodiesel é a palmeira de macaúba (Acrocomia aculeata) que apresenta grande potencial para esta aplicação devido a sua elevada produção de óleo e composição química favorável à produção de biodiesel. Isso se deve ao alto teor de ácido oléico presente no óleo derivado da polpa, podendo chegar de 66 - 70\% dependendo do clima e das características de solo onde a palmeira é cultivada (Hianne et al., 2005).

A síntese de biodiesel com base em fontes vegetais não comestíveis e óleos residuais têm sido desenvolvidos, entretanto a grande quantidade de ácidos graxos livres e umidade excessiva presentes nestas fontes requer o uso de um processamento adicional, aumentando o tempo reacional e consumindo muita energia. O emprego de processos e técnicas com o objetivo de fazer a síntese de biocombustíveis ser economicamente viável ganha espaço, e uma destas abordagens baseia-se no uso de reatores sonoquímicos (Stavarache et al., 2005).

O processo de produção enzimática de biodiesel em ultrassom vem como alternativa ao processo convencional empregando-se agitação mecânica, uma vez que as cavitações (formação, aumento e implosão de bolhas no meio reacional) geradas pelo ultrassom, aumentam a miscibilidade entre os reagentes, fornecem energia necessária para a reação, reduzem o tempo de reação e a quantidade de reagentes empregadas no processo, além disso, aumentam o rendimento e a seletividade da reação (Yu et al., 2010). Neste sentido o presente trabalho visa à produção de ésteres etílicos usando tecnologia de ultrassom na forma de banho e utilizando como substratos óleo derivado do fruto de macaúba (Acrocomia aculeata) e etanol em modos de produção batelada e contínuo, empregando como catalisador a lipase comercial Novozym 435.

\section{MATERIAL E MÉTODOS}

\subsection{Materiais}

Para a realização deste trabalho, nas reações de transesterificação utilizou-se como substratos óleo de macaúba (Acrocomia aculeata) sem nenhum tratamento prévio, álcool etílico (Merck, 99,9\% de pureza) e a enzima comercial Novozym 435, produzida a partir da lipase de Candida antarctica, imobilizada em resina acrílica macroporosa de troca iônica, pela Novozymes Brasil/ Araucária-PR. Os experimentos para produção enzimática de biodiesel em modos batelada e contínuo foram realizados em um banho de ultrassom (Unique ultrasonic Cleaner, modelo: USC-1800A, frequência US: 40KHz, potência US: 132W).

\subsection{Métodos}

Procedimento experimental: O experimentos para produção de ésteres etílicos derivados de óleo do fruto de macaúba em modos batelada e contínuo, foram realizados utilizando razão molar (óleo/ etanol) $1: 9,20 \%(\mathrm{~m} / \mathrm{m}$ ) de enzima Novozym 435 , em relação à massa total de substratos (óleo + etanol), temperatura $65^{\circ} \mathrm{C}$, potências ultrassônicas (0 Watts e 132 Watts), estas equivalentes a $0 \%$ e $100 \%$ da potência do equipamento e tempos reacionais variando até 360 minutos. Para o modo batelada os experimentos foram realizados em balão de $50 \mathrm{~mL}$ conforme Figura 1(a). 
No modo contínuo, além destas condições experimentais apresentadas anteriormente e tempo de reação até 66 minutos, o sistema experimental utilizado consistiu num reator de polietileno de alta densidade (com $1 / 16 \mathrm{~mm}$ de diâmetro externo, espessura de $1,1 \mathrm{~mm}$, diâmetro interno de $4,15 \mathrm{~mm}$, comprimento de 2 metros e volume total de $27 \mathrm{~mL}$ conforme apresentado na Figura 1(b) com vazão de alimentação de $2,5 \mathrm{~mL} / \mathrm{min}$ da mistura reacional óleo/etanol, e o reator empacotado com aproximadamente $11 \mathrm{~g}$ de catalisador, sendo este a enzima comercial Novozym 435. O tempo espacial da $1^{\text {a }}$ gota percorrido pela mistura reacional no reator ocorreu após 13 minutos de reação, sendo realizadas coletas de amostras para análise a cada 5,5 minutos.

Figura 1 - Reações de transesterificação em modos batelada (a) e contínuo (b).

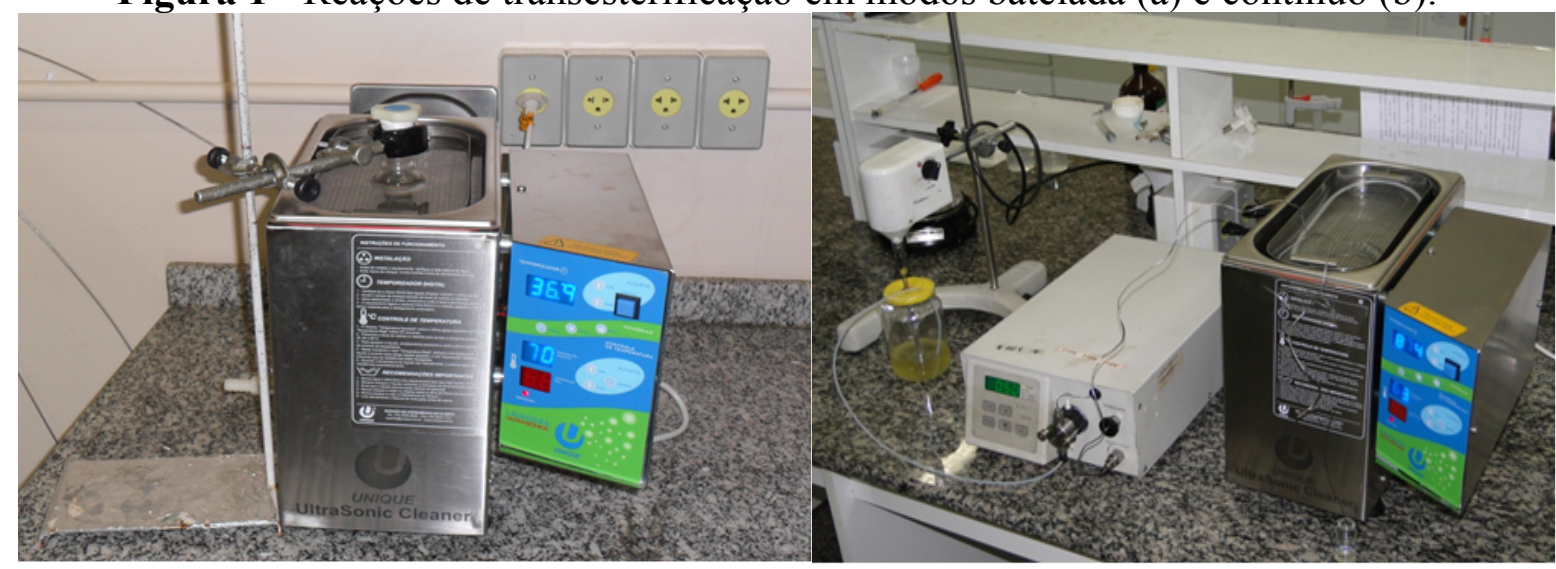

(a) Batelada

(b) Contínuo

A quantificação dos ésteres produzidos foi realizada por cromatografia gasosa (GCShimadzu 2010), nas condições cromatográficas descritas pela norma EN 14103 (2003), do Comitê Europeu para Padronizações. Conforme esta norma foi usado o padrão interno heptadecanoato de metila, com procedência Sigma-Aldrich e como solvente n- heptano (Sigma-Aldrich, 99,9\% de pureza). Após preparo da amostra, foi injetada $(1 \mu \mathrm{L})$ da mesma em um cromatógrafo gasoso (GC) (Shimadzu 2010), com injetor automático (Split) e detector de ionização de chama (FID). Utilizou-se a coluna capilar Rtx-WAX (30m x 0,25mm x 0,25 $\mu \mathrm{m})$ nas condições cromatográficas conforme norma descrita anteriormente.

\section{RESULTADOS E DISCUSSÃO}

Nas reações de transesterificação existem dois procedimentos básicos para a síntese de biodiesel, modo batelada e contínuo, que podem ser realizados em temperatura ambiente ou mais elevadas, à pressão atmosférica ou elevada, na presença ou ausência de catalisadores adequados e em sistemas com ou sem solventes orgânicos. No método batelada, as fases de reação e da separação são geralmente efetuadas no mesmo tanque, de modo que requer um maior volume do reator e tempos de reação e separação mais longos. Assim cabe ressaltar que quando se opera com processo em batelada, o controle da temperatura e da pressão da reação de forma independente é bastante difícil uma vez que a pressão do sistema é resultado da temperatura da reação (Thanh et al., 2010, Avellaneda e Salvadó, 2011). 
O processo contínuo proporciona um menor custo de produção, a qualidade do produto é uniforme, facilita o controle do processo e diminuindo o volume do reator, reduz-se o tempo de retenção necessário para atingir conversões desejadas (Veljkovic et al., 2012). Nas reações de transesterificação enzimática em banho de ultrassom foi avaliado o teor de ésteres etílicos produzidos, nas condições experimentais citadas anteriormente para os modos batelada e contínuo.

A fim de estudar a eficiência do ultrassom e realizar um comparativo entre os dois modos de produção de ésteres etílicos, realizaram-se quatro testes conforme apresentados nas Figuras 2 e 3. Através dos resultados obtidos, é possível verificar claramente que a potência ultrassônica apresenta influência positiva na produção de ésteres etílicos derivados de óleo de macaúba tanto para o modo de produção em batelada como contínuo. Percebe-se também que o uso do ultrassom, conduziu à maiores conversões em ésteres nos tempos cinéticos estudados quando comparados aos experimentos com apenas o uso da temperatura e $0 \%$ de potência ultrassônica.

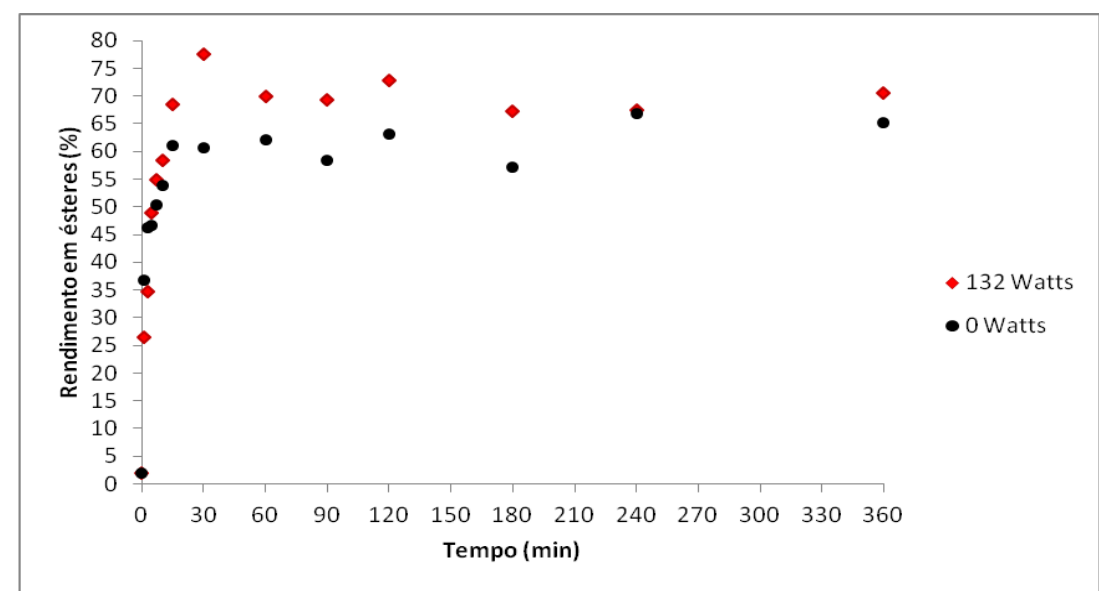

Figura 2 - Conversão em ésteres etílicos derivados de óleo de macaúba em modo batelada com potências ultrassônicas 0 Watts $(0 \%)$ e 132 Watts $(100 \%)$.

A tecnologia ultrassônica tem sido reconhecida como um método eficaz para aumentar a taxa de transferência de massa entre fases imiscíveis líquido-líquido no interior de um sistema heterogêneo. Por isso, esta técnica tem sido amplamente utilizada em várias reações químicas e biológicas para melhorar o rendimento dentro de um tempo de reação mais curto (Veljkovic et al., 2012).

Ao realizarmos uma comparação entre os dois modos (batelada e contínuo) verifica-se por meio das Figuras 2 e 3 que o modo batelada apresenta-se mais eficaz que o modo continuo sendo que para a produção de ésteres em modo batelada com o uso de potência ultrassônica (132 Watts) obtém-se conversões em ésteres de $78 \%$ em tempo reacional de 30 minutos. Em modo continuo a máxima conversão em ésteres obtida foi de $69 \%$ em 11 minutos de reação. Com o passar do tempo percebe-se que ocorre uma leve diminuição gradativa na conversão durante todo o processo chegando a aproximadamente $59 \%$ em ésteres após 60 minutos de reação. Este resultado provavelmente deve-se ao processo de redução da atividade catalítica da enzima, ocasionada pela incrustação do glicerol formado 
durante a reação, na superfície do suporte da lípase levando assim com o passar do tempo ao impedimento do acesso do substrato ao sitio ativo da enzima diminuindo sua atividade catalítica e consequentemente reduzindo o rendimento final em ésteres.

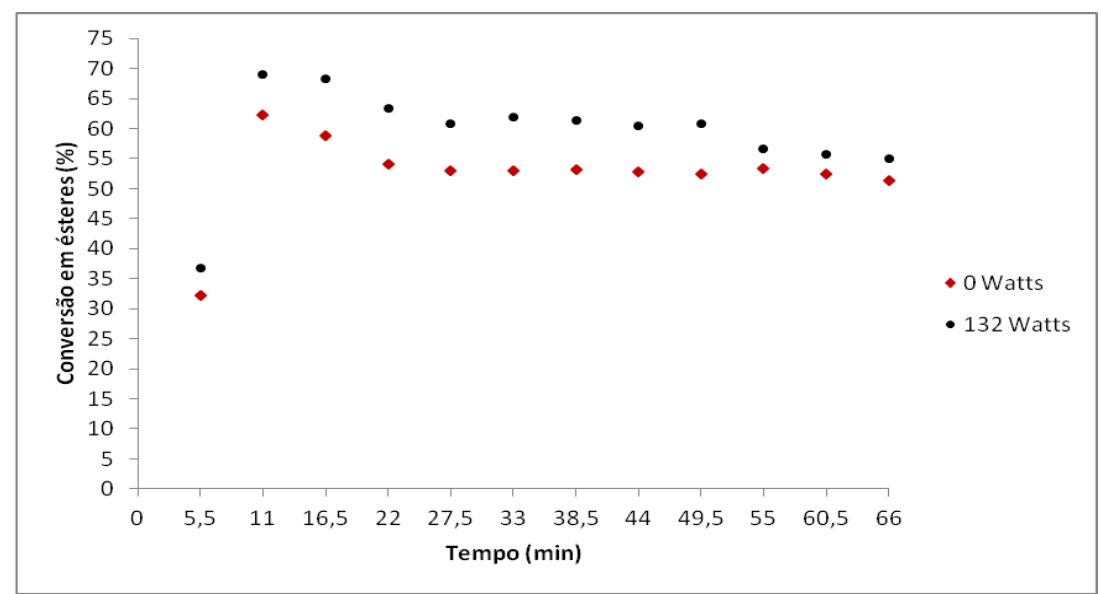

Figura 3 - Conversão em ésteres etílicos derivados de óleo de macaúba em modo contínuo com potências ultrassônicas 0 Watts $(0 \%)$ e 132 Watts $(100 \%)$.

Conforme citado no trabalho de Batistella (2011), na transesterificação de óleo de soja utilizando solvente orgânico (n-hexano) e por meio de sistema ultrassônico, houve uma tendência ascendente para os catalisadores (enzimas Lipozyme RM IM e Novozym 435). Ocorreu um aumento no rendimento nas primeiras 4 horas de reação (Lipozyme RM IM), rendimento de $55 \%$ e aproximadamente $37 \%$ (Novozym 435 ) nas condições experimentais (razão molar (1:3 óleo/etanol), temperatura $\left(65^{\circ} \mathrm{C}\right)$, concentração da enzima $(5 \%(\mathrm{~m} / \mathrm{m})$, sem adição de água e $100 \%$ de potência de ultrassônica $(37 \mathrm{kHz}$ e potência de $132 \mathrm{~W})$. A irradiação ultrassônica em reações catalisadas por enzimas provou ser um eficiente método para produção de biodiesel utilizando óleo de soja, etanol anidro. Apenas $10 \%$ da irradiação do ultrassom foram suficiente para atingir 99\% conversão de ésteres etílicos em 12 horas de reação (razão molar (1:4) de óleo de soja:etanol anidro, 5\% de lipase imobilizada LTL $(1: 1,5)$ (v/v) de hexano como solvente (óleo/solvente), $35^{\circ} \mathrm{C}$ a $\left.150 \mathrm{rpm}\right)(\mathrm{Ohe}, 2011)$.

\section{CONCLUSÕES}

Ao final do estudo percebe-se que a potência do ultrassom exerce influência positiva sobre a conversão em ésteres etílicos tanto nas reações em modo batelada, quanto contínuo mostrando-se assim como uma tecnologia de perspectiva promissora para o uso desta técnica em reações de transesterificação aplicando-se catálise enzimática. Ao comparar ambos os modos de reação de produção de ésteres estudados (batelada e continuo), pode-se concluir que o método batelada foi neste caso efetivamente melhor, com conversões em ésteres na ordem de $78 \%$ em período de tempo de 30 minutos. No modo continuo esses resultados mostraramse um pouco inferiores, (69\%), devido à diminuição da atividade catalítica da enzima ocasionada pela possível formação de glicerol dentro do reator contínuo, aderindo este sobre o suporte enzimático. 


\section{REFERENCIAS}

AVELLANEDA. F.; SALVADÓ. J. Continuous transesterification of biodiesel in a helicoidal reactor using recycled oil. Fuel Processing Technology. v. 92, 83-91, 2011.

BATISTELLA, L. Produção enzimática de biodiesel em solvente orgânico em banho de ultrassom. Dissertação de Mestrado. Universidade Regional Integrada do Alto Uruguai e das Missões - URI, Erechim, RS, Brasil, 2011.

BIODIESEL BR. (www.biodieselbr.com/biodiesel.htm) Acessado em 21 de novembro de 2013.

DEMIRBAS. A. Importance of biodiesel as transportation fuel. Energy Policy. v. 35, 46614670, 2007.

GOG, A.; ROMAN, M.; TOSA, M.; PAIZS, C.; IRIMIE, F. D. Biodiesel production using enzymatic transesterification - Current state and perspectives. Renewable Energy. v. 39, 10$16,2012$.

HIANE, P. A.; RAMOS FILHO, M. M.; RAMOS M. I. L.; MACEDO, M. L. R. Bocaiúva, Acrocomia aculeata (Jacq.) Lodd., Pulp and Kernel Oils: Characterization and Fatty Acid Composition. Brazilian Journal of Food Technology. v. 8, 256-259, 2005.

OHE, T. H. K. Produção de biodiesel etílico com uso de lipases extracelulares de fungos termofílicos. 2011.

STAVARACHE, C.; VINATORU, M.; NISHIMURA, R.; MAEDA, Y. Fatty acids methyl esters from vegetable oil by means of ultrasonic energy. Ultras. Sonochem. v. 12, 367-372, 2005.

THANH; L.T.; OKITSU; K.; SADANAGA; Y.; TAKENAKA; N.; MAEDA; Y.E.; BANDOW, H. A two-step continuous ultrasound assisted production of biodiesel fuel from waste cooking oils: A practical and economical approach to produce high quality biodiesel fuel. Bioresour. Technol. v. 101, 5394-5401, 2010.

YU, D.; TIAN, L.; WU, H.; WANG, S.; WANG, Y.; MA, D.; FANG, X. Ultrasonic irradiation with vibrationfor biodiesel production from soybean oil by Novozym 435. Process Biochemistry, v. 45, 519-525, 2010.

VELJKOVI'C, V.B.; AVRAMOVI'C, J.M.; STAMENKOVI'C, O.S. Biodiesel production by ultrasound-assisted transesterification: State of the art and the perspectives. Renew. Sustain. Energy Rev. v. 16, 1193-1209, 2012. 